\title{
High-Selectivity Single-Chip Spectrometer in Silicon for Operation at Visible Part of the Spectrum
}

\author{
José Higino Correia, Marian Bartek, Member, IEEE, and Reinoud F. Wolffenbuttel, Senior Member, IEEE
}

\begin{abstract}
A microspectrometer has been realized based on an array of Fabry-Perot optical thin-film filters. The 16-channel microspectrometer is compatible with IC fabrication methods and operates in the visible spectral range with an interchannel shift of $6 \mathrm{~nm}$. Each of the channels is sensitive in a single peak with full-width-half-maximum (FWHM) of $16 \mathrm{~nm}$. Also a FWHM below $\mathbf{2} \mathbf{~ n m}$ and finesse of $\mathbf{4 0}$ for narrow band operation is demonstrated. The device can easily be tuned during fabrication to cover a different spectral band only by adjusting the etching times without affecting the device layout. Such a device is extremely suitable for applications in microsystems because of its small size, high spectral selectivity, and low cost. Microspectrometers for the UV and IR regions are also feasible using this technique.
\end{abstract}

Index Terms-Array-type microspectrometer, Fabry-Perot etalon, finesse, FWHM, optical filter, visible light detector.

\section{INTRODUCTION}

C ONVENTIONAL benchtop spectrometers involve a complex system of lenses and moving parts (or a high-density photodetector array), and are thus bulky and expensive. By applying micromachining techniques, one may be able to produce a spectrometer with drastically reduced size and costs (in high-volume production). In addition, a number of these systems can be combined for improved spectral range and/or resolution. Moreover, an integrated optical device has a number of advantages over a conventional optical system, such as a simplified assembly, stable alignment, and compact size.

The dimensional advantage of a miniaturized spectrometer is, in many applications, of higher importance than its reduced resolution. For example, examination of products in a manufacturing line by means of laboratory analysis is time consuming, increases costs and sometimes stops production. Therefore, it has become important to perform online measurements in the process line in order to correct any process problems in real time. A small spectrometer offers a huge potential to serve the needs of future automated optical inspection systems.

Identification of the composition of gases and liquids, chemical analysis by optical absorption, emission-line characterization, colorimetry and biochemical analysis are some of the applications where a miniaturized spectrometer can be useful [1].

Manuscript received May 5, 1999; September 27, 1999. This work was supported in part by STW (Project DEL 55.3733), Delft University of Technology, and FCT-Portugal (Program Praxis XXI-BD/5181/95). The review of this paper was arranged by Editor K. Najafi.

J. H. Correia is with the Department of Industrial Electronics, University of Minho, 4800 Guimaraes, Portugal (e-mail: higino.correia@ dei.uminho.pt).

M. Bartek and R. F. Wolffenbuttel are with Delft University of Technology, ITS/Electrical Engineering, Laboratory, for Electronic Instrumentation/DIMES, 2628 CD, Delft, The Netherlands.

Publisher Item Identifier S 0018-9383(00)01944-4.
Previously developed microspectrometers [2]-[6], fabricated using bulk or surface micromachining, contain movable parts to perform wavelength tuning. As a result, they have limited reliability and are suitable only for operation in a limited spectral band (mostly near-IR) [6], [7]. Moreover, high-voltage electrostatic actuation is necessary for resonance cavity tuning.

Operation in the visible spectral range (with a single transmission peak) implies a maximum cavity length of $400 \mathrm{~nm}$. Fabrication and modulation of such a narrow airgap between the two mirrors is severally hindered by capillary forces inside of the cavity. Also, electrostatic pull-in and subsequent sticking of the two mirrors limits the operating range of the device to one third of the initial air gap [8]. Small resolution $(R=\lambda / \Delta \lambda$, with $\Delta \lambda$ the smallest wavelength difference distinguishable at a specific wavelength, $\lambda$ ) are a limitation of microspectrometers because the optical path length available is short (especially in grating-based microspectrometers) compared with the large dimensions systems.

\section{MicrospeCtrometer Design}

\section{A. Complete Structure}

A new single-chip, Fabry-Perot array-type microspectrometer was developed. This device is suitable for operation in the visible spectral range and does not require electrostatic actuation. The Fabry-Perot structure is a simple interferometer where the incident light suffers multiple reflections between the coated surfaces that define the cavity. The emerging wavefronts interfere constructively only if there is no phase difference between them. At other wavelengths, destructive interference of the transmitted wavefronts reduces the transmitted intensity toward to zero. Therefore, this device acts as a filter that transmits certain wavelengths and reflects the others back to the light source.

The realized microspectrometer, shown schematically in Fig. 1 (an individual channel in cross-section) and Fig. 2 (the complete $4 \times 4$ array microspectrometer), is fully compatible with standard IC processing methods.

The impinging spectrum is filtered in the Fabry-Perot resonator and the intensity of the selected spectral component is measured in transmission using an underlying integrated photodiode array. On top of each photodiode a deposited $\mathrm{Al} / \mathrm{SiO}_{2} / \mathrm{Ag}$ layer stack functions as a Fabry-Perot optical filter. The thickness of the PECVD-silicon-dioxide layer, which is enclosed between two semitransparent metallic mirrors, determines the wavelength tuned to. In $N$ subsequent plasma etching steps (using different photoresist masks), the initially deposited PECVD oxide layer is thinned forming $2^{N}$ channels, 


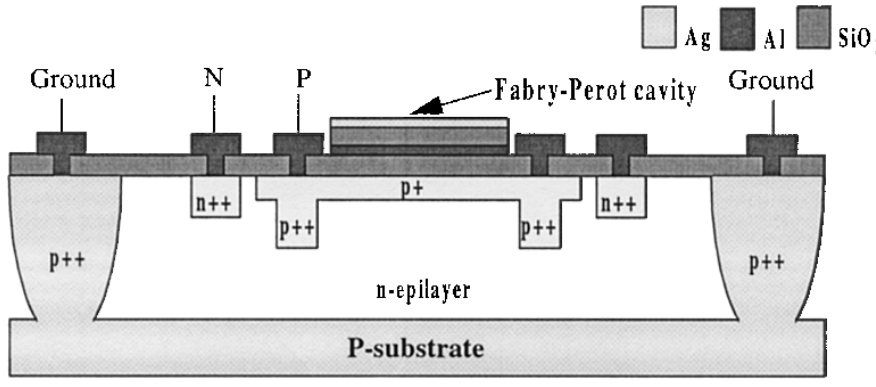

Fig. 1. Schematic structure of the microspectrometer. One individual channel is shown in cross section.

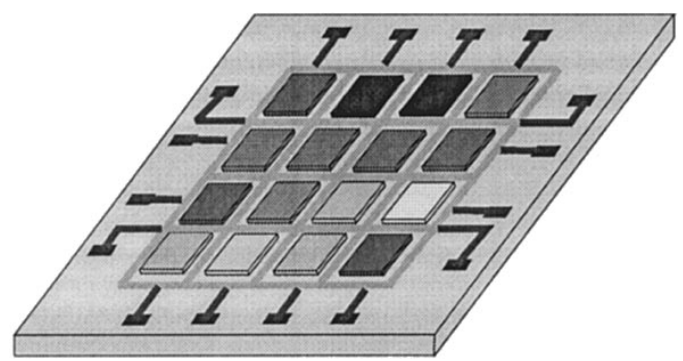

Fig. 2. A $4 \times 4$ array microspectrometer in perspective. Each of the Fabry-Perot cavities is tuned to transmit in different spectral band.

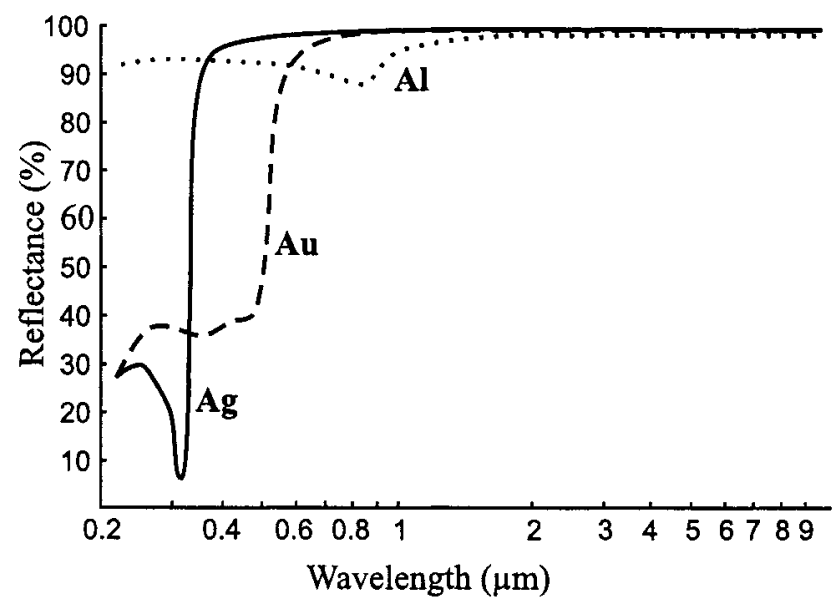

Fig. 3. Reflectance of $\mathrm{Ag}, \mathrm{Au}$, and $\mathrm{Al}$ as a function of radiation wavelength [10].

each with a different resonance cavity length. The oxide passivation layer between the lower mirror of the cavity and the photodiode introduces a wavelength-dependent transmission of the incident radiation. Its thickness was designed to yield a flat transmittance over the visible spectral range (approximately $50 \mathrm{~nm}$ ) [9], [10]. Evaporated metallic mirrors were used instead of high-performance dielectric mirrors, to maintain fabrication simplicity (only one layer must be deposited).

Another advantage of metallic mirrors is the suitability for use over a wide spectral range [11]. Silver and aluminum have been selected for high reflectivity at visible wavelengths (see Fig. 3). Fabry-Perot filters using metallic mirrors cannot provide both high finesse (ratio between the free spectral range and FWHM) and high transmittance simultaneously due to the optical absorption in the metal layers (Fig. 4). Unlike macroscopic

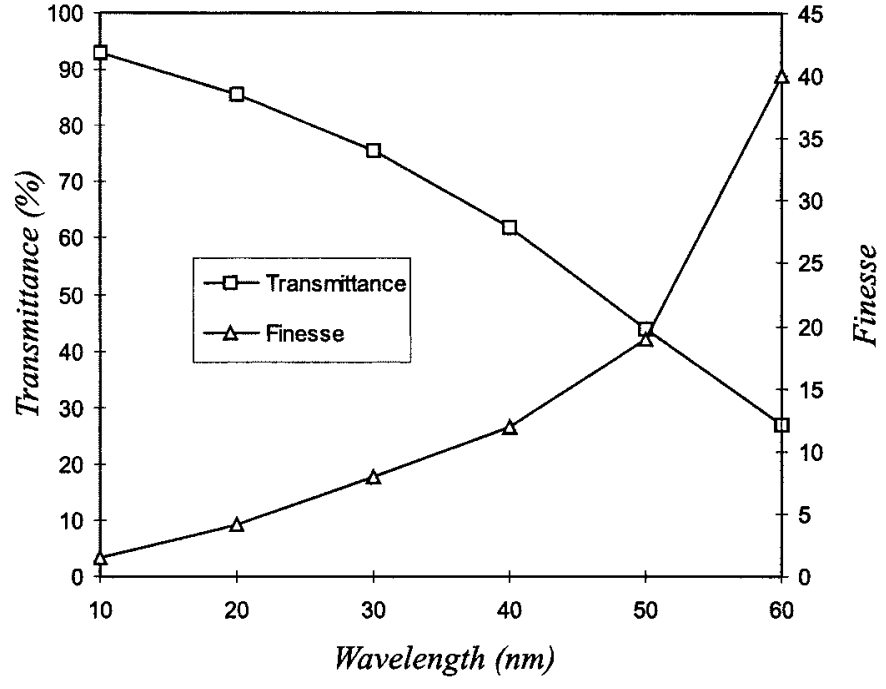

Fig. 4. Transmittance and finesse as a function of silver thickness.

applications of silver-based reflective coatings [12], the poor environmental resistance of silver is not critical in a microsystem application. Sealing of a complete system avoids any environmentally induced mirror degradation (a transparent package, e.g. glass).

\section{B. Selection of Materials}

According to the simulation results, the best option for a Fabry-Perot resonance cavity for the visible range in terms of optical characteristics is to use silver mirrors (higher reflectance than aluminum mirrors) with an intermediate air gap (lowest refractive index). Because the array type has fixed cavities of different widths, an array of Fabry-Perot etalons (cavity medium different from air) was implemented. In conventional devices, it is common to use a dielectric film such as glass or silica as cavity medium. Two different dielectric materials were investigated: silicon nitride $\left(\mathrm{Si}_{x} \mathrm{~N}_{y}\right)$ and PECVD silicon dioxide $\left(\mathrm{SiO}_{2}\right) . \mathrm{Si}_{x} \mathrm{~N}_{y}$ films are characterized by stronger dispersion and larger values of the refractive index. The dependence of refractive index on the wavelength for the PECVD $\mathrm{SiO}_{2}$ films is more constant (varies from 1.47 to 1.46 between $400 \mathrm{~nm}$ to $800 \mathrm{~nm}$ wavelengths) [9]. Therefore, $\mathrm{PECVD} \mathrm{SiO}_{2}$ was selected.

Due to fabrication constraints, the bottom mirror of the etalon is a thin aluminum film instead of silver (an unsuitable metal for subsequent oxide deposition). Only the top mirror is a thin film of silver deposited at the last step in the fabrication process. The compatibility with standard microelectronics processing implies that only $\mathrm{Si}$-compatible materials should be used, avoiding contamination of the process with the deposition of silver in an intermediate step.

\section{Optical Simulations of the Fabry-Perot Etalon}

A thin-film optics software package, TFCalc v.3.2 (from Software Spectra), was used for structural optimization of the Fabry-Perot filter. The transmittance of a $60 \mathrm{~nm}-\mathrm{Ag} / 1000$ $\mathrm{nm}-\mathrm{SiO}_{2} / 60 \mathrm{~nm}-\mathrm{Ag}$ layer stack (Fig. 5) shows a FWHM of $1.8 \mathrm{~nm}$ and a finesse of 40 . The Ag-layer thickness is a tradeoff between achievable FWHM and absorption loss. When 
aluminum is used for the bottom mirror (due to fabrication constraints), the performance slightly decreases due to higher absorption and less reflectance of aluminum films compared to that of silver films.

\section{Phototransducers}

Each channel in the microspectrometer is composed of a Fabry-Perot etalon with an optical detector underneath. The detector is a p-n junction photodiode fabricated in a standard bipolar process (see Fig. 1). The absorption of light in silicon is wavelength dependent: long wavelength light penetrates much deeper before being absorbed.

Junction isolation using deep boron diffusions electrically separates individual photodiodes (see Fig. 1). The top surface between different channels is covered with metal in order to shield from stray light.

Limiting factors in the opto-electrical conversion in a photodiode are the size and uniformity of the effective sensing area and dark current. Dark current is the current that flows in a photodiode when there is no optical radiation incident on the photodiode. Compensation techniques are usually employed to minimize this effect. As the dark current is temperature dependent, one measurement at the beginning of the experiment is usually not sufficient. A dark-current-compensation channel is implemented using a photodiode completely covered with metal.

The dominant noise source in a photodiode is shot noise [13]. Another form of noise is the fixed pattern noise. It is defined as the photoresponse variation between adjacent photodiodes. The fixed pattern noise is controlled by the size of the photodiode relative to the minimum lithographic feature size. As the dimensions of the photodiodes $\left(1 \mathrm{~mm}^{2}\right)$ are relatively large, the fixed pattern noise is negligible [14], less than $1 \%$ in our case.

The cross-talk resulting from photon-produced minority-carrier diffusion is a problem in optical detection with more than one channel. The carriers generated outside the junction depletion layer may diffuse to a neighboring photodiode and thus introduce an undesired current component. In certain silicon processes the diffusion length of such carriers can be up to $100 \mu \mathrm{m}$ [15]. So, this minority-carrier diffusion causes cross-talk in a closely-spaced photodiode array. Moreover, the problem cannot be resolved by a light shield, because the carriers are primarily produced under a photodiode. The solution is to form a deep p-diffusion that contacts the p-substrate and is connected to ground potential (junction isolation). In this way, the photocurrent in a diode is contributed to only by the photocarriers generated in or near the depletion layer between the p-diffusion and the n-diffusion regions. Minority carriers generated above or below the n-diffusion/p-substrate junction or diffusing from neighboring photodiodes, will be swept away from the n-diffusion region by the junction field, or be bypassed to the power supply.

\section{DEVICE FABRICATION}

The photodiode array was fabricated using a bipolar process in silicon [16]. The Fabry-Perot etalons were added in a postprocess module. The postprocess module, which was used to

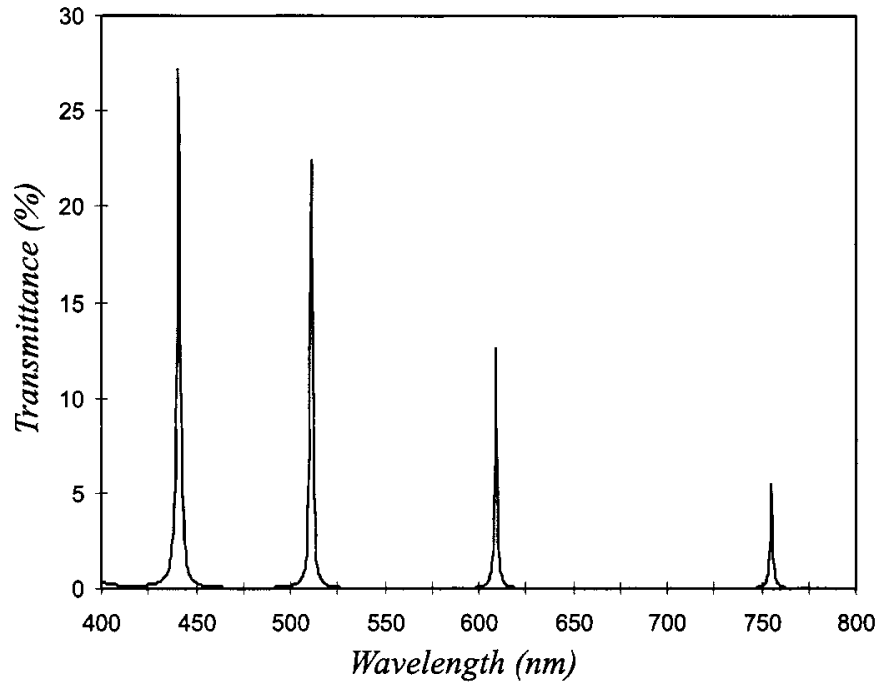

Fig. 5. Simulated transmittance versus wavelengths for $60 \mathrm{~nm} \mathrm{Ag/1000}$ $\mathrm{nm} \mathrm{SiO}_{2} / 60 \mathrm{~nm} \mathrm{Al}$ layer stack. Fourth-order mode of operation (four peaks achieved). The different values of transmittance result from the optical properties of the materials used.

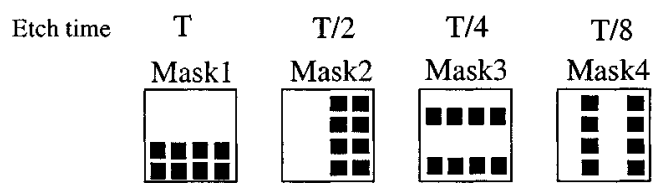

Fig. 6. Principle used for fabricating a $4 \times 4$ array microspectrometer. After the deposition of the silicon dioxide, each mask is used with a different etch time. In this example the first mask will etch one half of whole thickness, the second one fourth, etc.

fabricate the 16 etalons, each with a different thickness, requires further explanation. The formation of the Fabry-Perot etalon starts with the deposition of a $20 \mathrm{~nm} \mathrm{Al} \mathrm{layer} \mathrm{after} \mathrm{completion}$ of the bipolar process (including metallization and thinning of the oxide above the photodiodes). The oxide passivation layer on top of the photodetector (between the Fabry-Perot etalon and $\mathrm{Si}$ substrate surface) is thinned to reduce its influence on the spectral response. The thin Al layer is evaporated and patterned using liftoff. Subsequently, a PECVD oxide layer is deposited with a thickness equal to the maximum cavity length (300 $\mathrm{nm}$ in this example). The thickness of the PECVD silicon dioxide layer, which is enclosed between two semitransparent metallic mirrors, determines the wavelength for tuning. In $N$ subsequent plasma etching steps (for which different photoresist masks are used), the initially deposited PECVD oxide layer is thinned that $2^{N}$ channels are formed, each with a different resonance cavity length. An example with four masks for a $4 \times 4$ array-type microspectrometer is shown in Fig. 6. After the deposition of the silicon dioxide, each mask used has a different etching time $(T, T / 2, T / 4, T / 8)$. A relative thickness uniformity better than $2 \mathrm{~nm}$ was achieved between cavity lengths. A silver layer is deposited at the very end of the fabrication sequence and patterned using lift-off. Fig. 7 presents a complete description of the bipolar post-processing fabrication sequence. Fig. 8 shows a SEM photograph presenting the cross section of one of the channels. Fig. 9 shows a photograph of the fabricated device with overall dimensions of $4.7 \times 4.7 \mathrm{~mm}^{2}$. 


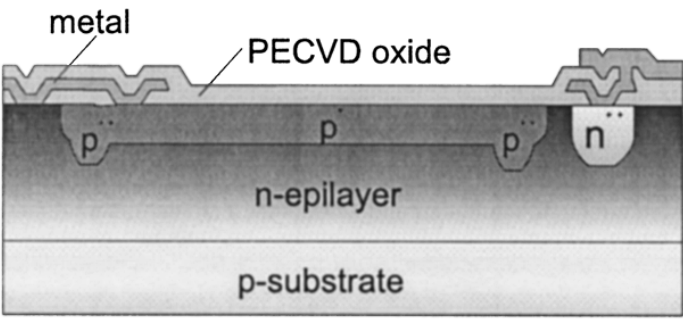

(a) Completed bipolar process.

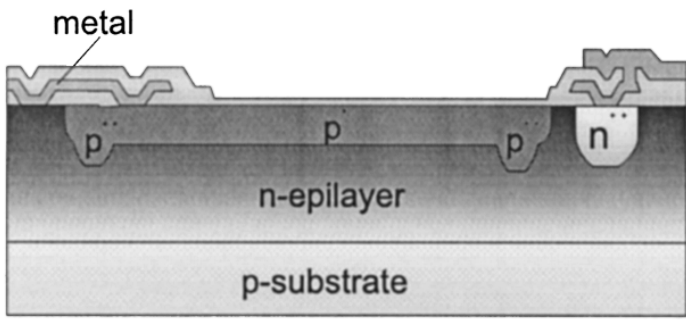

(b) Thinning of the oxide above photodiode active area.

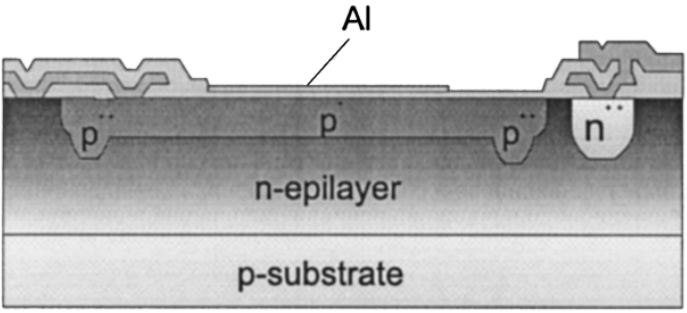

(c) Deposition of lower aluminum mirror by means of lift-off.

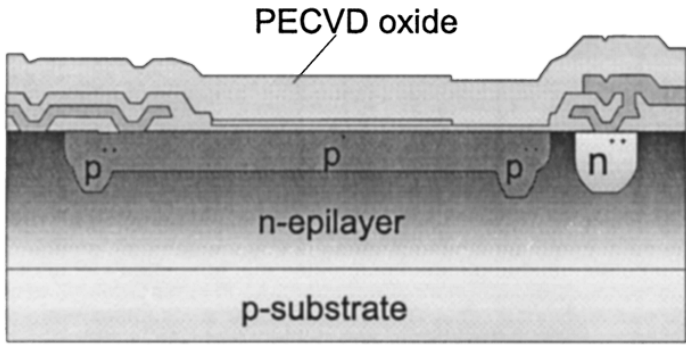

(d) PECVD oxide deposition and sequential thinning.

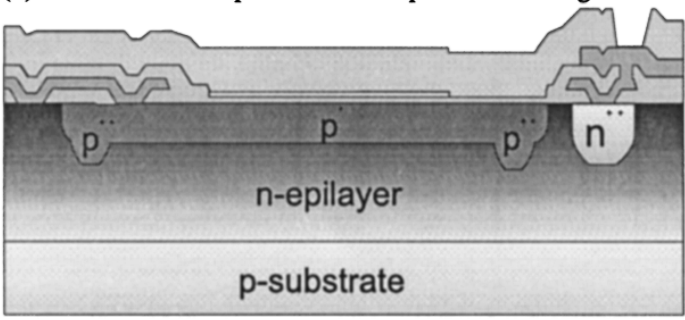

(e) Bond pad opening.

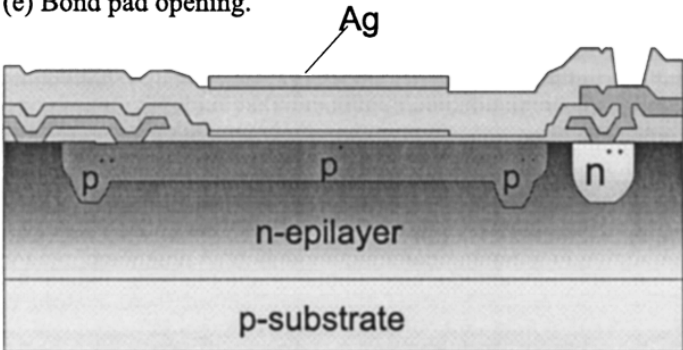

(f) Deposition of upper Ag mirror by means of lift-off (not included in the standard processing line).

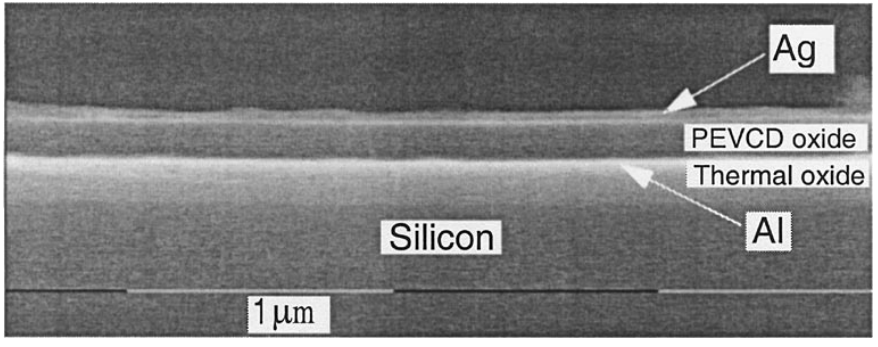

Fig. 8. SEM photograph showing the cross-section of one of the channels

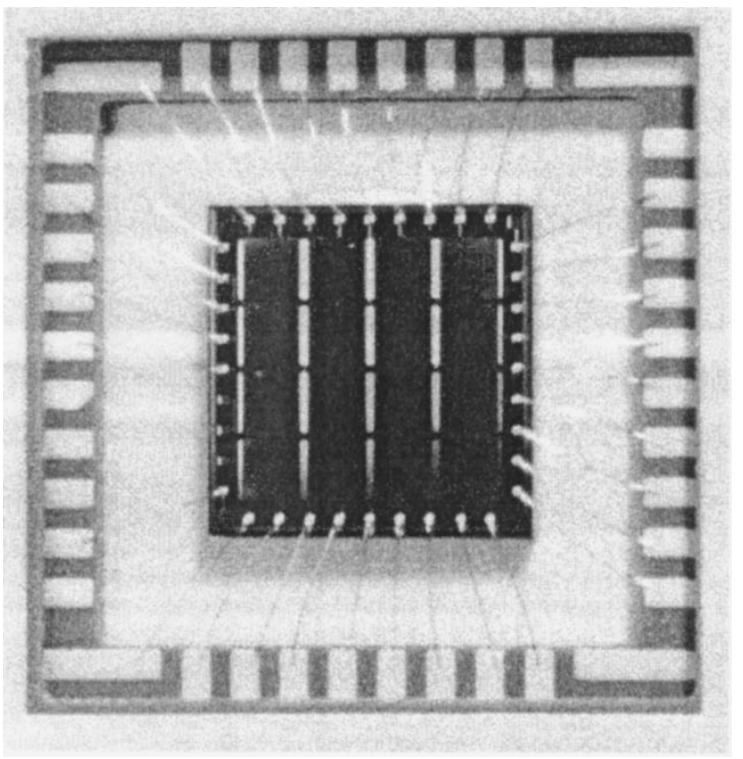

Fig. 9. Photograph of the fabricated microspectrometer with $4 \times 4$ channels. The die area is $4.7 \times 4.7 \mathrm{~mm}^{2}$.

\section{EXPERIMENTAL RESULTS}

The electrical characteristics and spectral responsivity were measured using an HP4142B DC Source/Monitor (full-scale range from $10^{-15} \mathrm{~A}$ to $1 \mathrm{~A}$ and a resolution of $10^{-13} \mathrm{~A}$ ). A $100 \mathrm{~W}$ quartz tungsten halogen lamp with a monochromator TRIAX-180 (1200 g/mm grating with a spectral dispersion of $3.6 \mathrm{~nm} / \mathrm{mm}$ and a spectral resolution of $0.3 \mathrm{~nm}$ at $546 \mathrm{~nm}$ ), was used as light source. A collimator lens was used to image the light on the entrance slit. At the exit slit, a pinhole and a focusing lens were used to achieve a beam with a diameter of about $400 \mu \mathrm{m}$. The monochromator, optical hardware and the microspectrometer were assembled and aligned on an optical table. Without focusing lens, the output beam coming from the monochromator illuminates the complete etalons array. Using the pinhole, focusing lens and controlling the distance to the microspectrometer it is possible to illuminate only one etalon. A translation mount was used for exact positioning of the microspectrometer in the vertical, horizontal and depth planes in order to scan all etalons. The measurements were calibrated with a commercially available photodiode, Hamamatsu S1336-5BQ.

System operation is demonstrated in a limited range using a 16-channel micro-spectrometer designed for operation in the spectral range between 400 to $500 \mathrm{~nm}$ with interchannel

Fig. 7. Post bipolar processing fabrication sequence. 


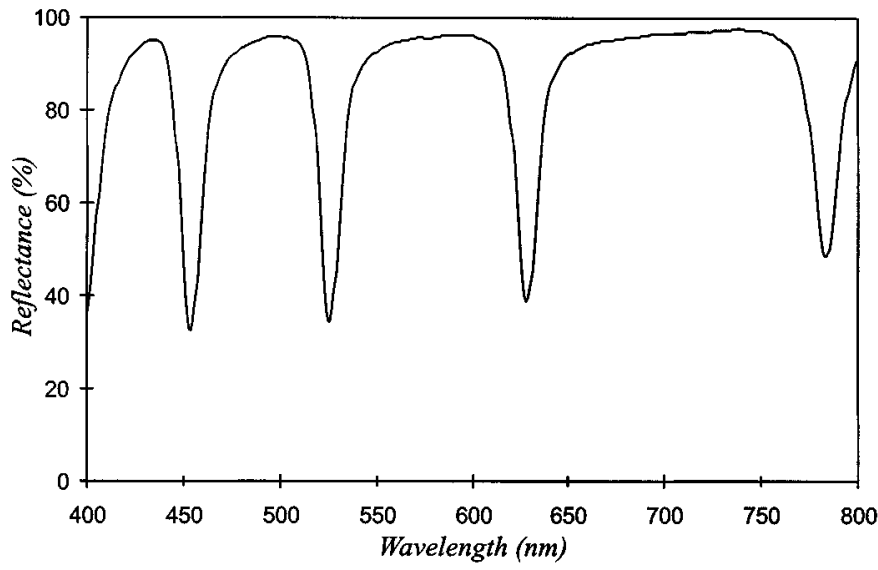

Fig. 10. Measured reflectance vs. wavelength for a $45 \mathrm{~nm} \mathrm{Ag/1000} \mathrm{nm}$ $\mathrm{SiO}_{2} / 20 \mathrm{~nm} \mathrm{Al}$ layer stack.

TABLE I

ELECTRICAL AND OPTICAL CHARACTERISTICS

\begin{tabular}{l|l}
\hline \multicolumn{1}{c|}{ Feature } & \multicolumn{1}{c}{ Result } \\
\hline Technology & Bipolar \\
\hline Device area & $4.7 \times 4.7 \mathrm{~mm}^{2}$ \\
\hline $\begin{array}{l}\text { FWHM } \\
@ \mathrm{t}_{\mathrm{SiO} 2}=300 \mathrm{~nm}\end{array}$ & $16 \mathrm{~nm}$ \\
\hline $\begin{array}{l}\text { Finesse } \\
@ \mathrm{t}_{\mathrm{SiO} 2}=300 \mathrm{~nm}\end{array}$ & Single peak \\
\hline $\begin{array}{l}\text { FWHM (simulated) } \\
@ 60 \mathrm{~nm} \text { Ag mirrors }\end{array}$ & $1.8 \mathrm{~nm}$ \\
$@ \mathrm{t}_{\mathrm{SiO} 2}=1 \mu \mathrm{m}$ & \\
\hline $\begin{array}{l}\text { Finesse (simulated) } \\
@ 60 \mathrm{~nm} \text { Ag mirrors }\end{array}$ & 40 \\
$@ \mathrm{t}_{\mathrm{SiO} 2}=1 \mu \mathrm{m}$ & \\
\hline Dark current@ 0 V & $0.2 \mathrm{pA}$ \\
\hline Dark current@ -4 V & $1 \mathrm{pA}$ \\
\hline $\begin{array}{l}\text { Spectral response } \\
\text { maximum @-4V }\end{array}$ & $0.013 \mathrm{AW} @ 388 \mathrm{~nm}$ \\
\hline \begin{tabular}{l} 
Incident angle range \\
\hline
\end{tabular} & $\pm 10^{\circ}$ \\
\hline
\end{tabular}

shift of about $6 \mathrm{~nm}$. Each of the channels consists of a $20 \mathrm{~nm}-\mathrm{Al} / \mathrm{SiO}_{2} / 45 \mathrm{~nm}-\mathrm{Ag}$ layer stack, where the oxide layer thickness changes between $225 \mathrm{~nm}$ and $300 \mathrm{~nm}$ with $5 \mathrm{~nm}$ steps.

Optical spectral measurements in reflectance (using an external detector, Leica Microscope Photometer System) show that each of the channels is sensitive to only one narrow spectral band. Independent measurements were performed on a cavity of thickness $1000 \mathrm{~nm}$. These results indicate a FWHM of $6 \mathrm{~nm}$ and a finesse of 12 (see Fig. 10).

Subsequently, measurements were performed on the device with integrated photodiodes. A dark current of $0.2 \mathrm{pA}$ at $0 \mathrm{~V}$ and $1 \mathrm{pA}$ at $-4 \mathrm{~V}$ (see Table I) was measured for a square photodiode with an active area of $1 \mathrm{~mm}^{2}$. The photocurrent measured in a normal channel at the same conditions is $10 \mu \mathrm{A}$ at

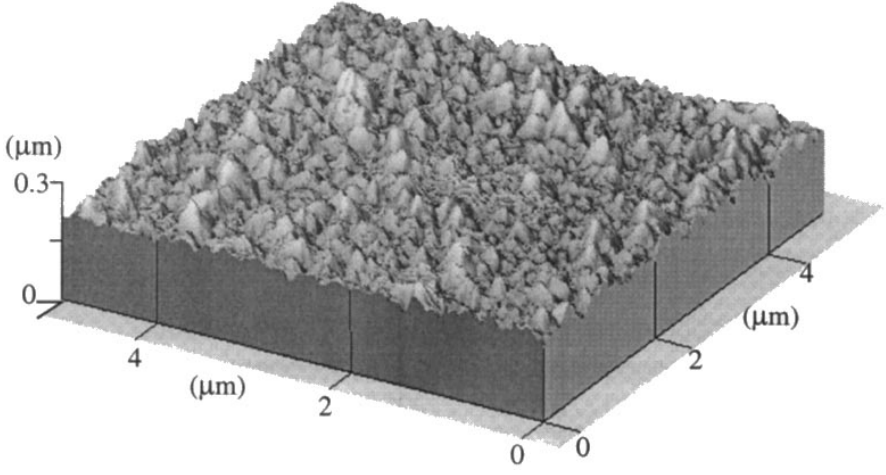

Fig. 11. AFM surface scan of a $45 \mathrm{~nm} \mathrm{Ag/300} \mathrm{nm} \mathrm{PECVD} \mathrm{oxide/} 20 \mathrm{~nm} \mathrm{Al} / 250$ thermal-oxide layer stack (array-type device mirror surface).

$-4 \mathrm{~V}$. The dark current directly limits the signal to noise ratio (SNR). SNR takes into account the noise due to: photons (the signal entering the detector has an intrinsic photon noise), photoelectrons (the photoelectric effect converts the photons into photoelectrons with some quantum efficiency), gain (internal gain of the photodetector, it increases both the signal and the noise) and receiver circuit (when the current is collected). The photocurrent, at a given source spectral power, is the result of a tradeoff between sensitivity, selectivity, and the number of channels used. An increased Ag-layer thickness improves selectivity but causes higher absorption losses and low sensitivity and thus decreases SNR. The presence of the Fabry-Perot filter limits the light transmitted to the underlying photodiode to less than $15 \%$ of the incident light.

An atomic force microscope (AFM) was employed to examine the surface morphology of the realized Fabry-Perot structures. The RMS roughness of the surface mirror in the array-type microspectrometer is typically $15 \mathrm{~nm}$ (see Fig. 11). A possible explanation for the high value can be the back etching applied to the PECVD oxide in order to define the cavity gap.

Fig. 12 presents the spectral responsivity (A/W) between $400 \mathrm{~nm}$ to $800 \mathrm{~nm}$ for all 16 channels using on-chip photodiodes. The ratio between the base line and the peak maximum ranges from four to seven. The relatively high stray light, beam divergence and the roughness surface are responsible for the background signal. Stray-light compensation methods must be used in order to compensate for the nonidealities of both the incident light beam and the Fabry-Perot etalon.

A solution to this problem is to use a compensation structure [17]. It consists of the same layer stack as used in any of the active channels. The difference is that the optical length of the cavity is decreased below $\lambda / 10(<40 \mathrm{~nm}$ if applied for measurements in the visible spectral range). This excludes any resonance inside the cavity. However, the parasitic signal caused by stray-light transmittance is similar to that of the active channel and can be used for compensation. Photodiodes are integrated underneath both the active and the compensating device and after subtraction of the photocurrents a compensated signal results. It should be mentioned that the conventionally used dark current compensation (an opaque layer deposited on top of a photodetector) compensates only for the nonidealities (dark current) of the detector itself. This method, in contrary, compensates for the nonidealities of detector, Fabry-Perot filter 


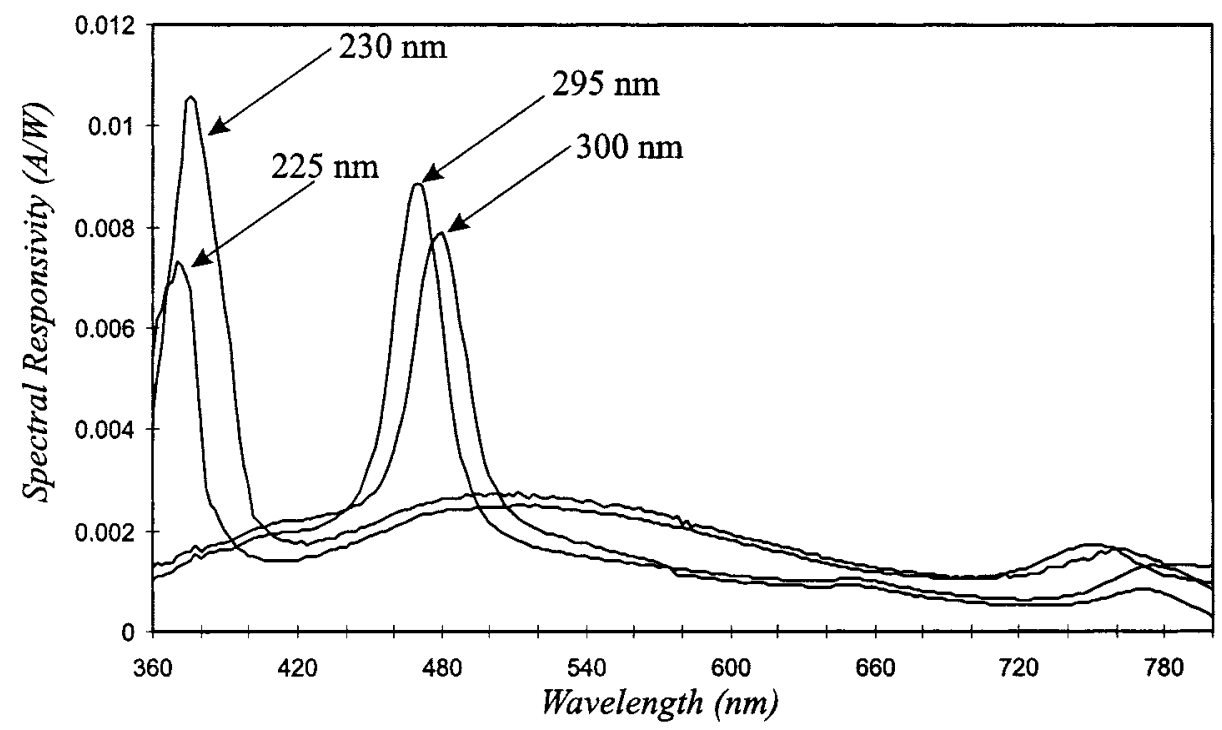

Fig. 12. Spectral responsivity of some channels of the 16-channel microspectrometer for a $45 \mathrm{~nm} \mathrm{Ag} / \mathrm{SiO}_{2} / 20 \mathrm{~nm} \mathrm{Al} \mathrm{layer} \mathrm{stack.} \mathrm{The} \mathrm{SiO}{ }_{2}$ layer thickness is used as a parameter and changes from $225 \mathrm{~nm}$ to $300 \mathrm{~nm}$ in $5 \mathrm{~nm}$ increments.

TABLE II

ARray-Type SPeCtrometers PERformance COMPARISON

\begin{tabular}{c|c|c|c|c|c}
\hline & $\begin{array}{c}\text { FWHM } \\
(\mathrm{nm})\end{array}$ & $\begin{array}{c}\text { FSR } \\
(\mathrm{nm})\end{array}$ & $\begin{array}{c}\text { Accuracy } \\
(\mathrm{nm})\end{array}$ & Resolution & $\begin{array}{c}\text { Dispersive } \\
\text { element }\end{array}$ \\
\hline $\begin{array}{c}\text { Array type } \\
\text { (this work) }\end{array}$ & 16 & $\begin{array}{c}400-520 \\
400-472\end{array}$ & \pm 3 & $\begin{array}{c}25^{*} \\
222^{* *}\end{array}$ & FP etalon \\
\hline $\begin{array}{c}\text { Low-cost } \\
\text { Spectr.[15] }\end{array}$ & $9.1 @ 632.8$ & $450-750$ & 2.55 & 69.8 & Grating \\
\hline $\begin{array}{c}\text { Spectronic } \\
20[16]\end{array}$ & 20 & $340-950$ & \pm 2.5 & $\begin{array}{c}47.5 \\
@ 950 \mathrm{~nm}\end{array}$ & Grating \\
\hline
\end{tabular}

${ }^{k}$ First mode operation (single-peak). ${ }^{* *}$ Fourth mode operation (four peaks) and $\mathrm{R}=\lambda / \mathrm{FWHM}$

and incident light beam at the same time. After photocurrent subtraction, the average background signal level is decreased by a factor of ten and, as a consequence, the spectral selectivity of the device is increased.

\section{CONCLUSION}

A single-chip spectrometer, using Fabry-Perot resonance cavities on a distributed photodetectors array, was fabricated and characterized (see Table I). The advantage of the device presented is that it can easily be tuned during fabrication to cover different spectral bands, by adjusting the etching time only, without affecting the device layout. Such a device is extremely suitable for applications in microsystems, because of its small size, high spectral selectivity, and low cost. Microspectrometers for the UV and IR are also feasible with this technique using dielectric mirrors.

The performance of the array-type microspectrometer presented is compared with that of a low-cost spectrometer [18] and with a commercially available spectrometer, (Spectronic 20) [19] (see Table II). Both spectrometers are based on a grating element and use a CCD detector. The free spectral range in the array-type device is limited by the low quantum efficiency of the photodiodes at low wavelengths. The high resolution in Fabry-Perot based spectrometers is obtained for high interference orders. In our case a resolution of 222 is achieved in fourth mode (four peaks obtained). But for a single peak, first mode, a reasonable resolution of 25 was measured.

The technique used for fabrication of the Fabry-Perot etalons allows, for simple expansion of the number of channels to 64 or 128 , to increase the operation range in the visible part of the spectrum. The minimum value reproducible and controllable in the difference at the $\mathrm{SiO}_{2}$ film thickness between two different channels is $2 \mathrm{~nm}$ using the RIE process to etch the $\mathrm{SiO}_{2}$ layer.

The reduction of the active channel area will increase the cross-talk between detectors, in order to minimize it we are considering to use silicon-on-insulator (SOI) wafers in future.

\section{ACKNOWLEDGMENT}

The authors would like to thank the staff of Delft Institute of Microelectronics and Submicron Technology (DIMES), especially J. Groeneweg, for technical assistance in fabrication of the devices. 


\section{REFERENCES}

[1] J. H. Correia, M. Bartek, and R. F. Wolffenbuttel, "High-selectivity single-chip spectrometer for operation at visible wavelengths," in IEDM Tech. Dig., San Francisco, CA, 1998, pp. 467-470.

[2] J. D. Patterson and B. van Zeghbroeck, "Fabrication and analysis of $\mathrm{Si} / \mathrm{SiO}_{2}$ micro-mechanical modulators," in Dig. IEEE/LEOS 1996 Summer Topical Meeting on Optical MEMS and Their Applications, 1996, pp. 25-26.

[3] T. A. Kwa, P. M. Sarro, and R. F. Wolffenbuttel, "Backside-illuminated silicon photodiode array for an integrated spectrometer," IEEE Trans. Electron Devices, vol. 44, pp. 761-765, May 1997.

[4] N. F. Raley et al., "A Fabry-Perot microinterferometer for visible wavelength," in Proc. 5th IEEE Solid State Sensor and Actuator Workshop, Hilton Head Island, SC, 1992, pp. 170-173.

[5] J. H. Correia, M. Bartek, and R. F. Wolffenbuttel, "Bulk-micromachined tunable Fabry-Perot microinterferometer for the visible spectral range," in Proc. Eurosensors XII, Southampton, U.K., Sept. 1998, pp. 287-290.

[6] A. T. T. D. Tran, Y. H. Lo, Z. H. Zhu, D. Haronian, and E. Mozdy, "Surface micromachined Fabry-Perot tunable filter," IEEE Photon. Technol. Lett., vol. 8, pp. 393-395, Mar. 1996.

[7] R. T. Carline, D. A. O. Hope, D. J. Robbins, and M. B. Stanaway, "Vertical cavity longwave infrared $\mathrm{SiGe/Si}$ photodetector using a buried silicide mirror," in IEDM Tech. Dig., Washington, DC, 1997.

[8] S. D. Senturia, N. Aluru, and J. White, "Simulating the behavior of MEMS devices: Computational methods and needs," IEEE Comput. Sci. Eng., pp. 30-43, Mar. 1997.

[9] D. P. Poenar and R. F. Wolffenbuttel, "Optical properties of thin-film silicon-compatible materials," Appl. Opt., vol. 36, pp. 5122-5128, July 1997.

[10] R. F. Wolffenbuttel, "Photodiodes in silicon with an electrically-programmable UV response," Sens. Actuators A, vol. 22, pp. 559-563, 1990.

[11] H. A. Macleod, Thin-Film Optical Filters, 2nd ed. Bristol, U.K.: Adam Hilger, 1986.

[12] D.-Y. Song, R. W. Sprague, H. A. Macleod, and M. Jacobson, "Progress in the development of a durable silver-based high-reflectance coating for astronomical telescopes," Appl. Opt., vol. 24, pp. 1164-1170, 1985.

[13] C. Decusatis, Handbook of Applied Photometry. Philadelphia, PA: AIP, 1997.

[14] B. Fowler, "CMOS area image sensors with pixel level A/D conversion," Ph.D. dissertation, Stanford Univ., Stanford, CA, 1995.

[15] C. Wang, Y. Ni, and F. Devos, "A spatio-temporal differentiation light sensor," Sens. Actuators A, vol. 62, pp. 492-495, 1997.

[16] R. S. Muller and T. I. Kamis, Device Electronics for Integrated Circuits, New York: Wiley, 1986.

[17] M. Bartek, J. H. Correia, S. H. Kong, and R. F. Wolffenbuttel, "Straylight compensation in thin-film Fabry-Perot optical filters: Application to an on-chip spectrometer," in Proc. Transducers'99, Sendai, Japan, June 7-10, 1999, pp. 240-243.

[18] G. M. Yee et al., "Miniature spectrometers for biochemical analysis," Sens. Actuators A, vol. 58, pp. 61-66, 1997.

[19] Data Catalog. Rochester, NY: Milton Roy/Spectronic Instruments Inc., 1997.

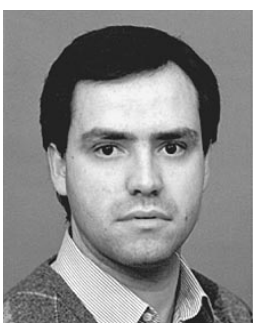

José Higino Correia (S'96-M'00) received the B. S. degree from the University of Coimbra, Portugal, in 1990, the PAPCC (equivalent to M.Sc. degree) in electronics and instrumentation in 1994 from the Department of Industrial Electronics, University of Minho, Portugal, and the Ph.D. degree in the field of microsystems for optical spectral analysis, in 1999, from Delft University of Technology, The Netherlands.

Since May 1999, he has been an Auxiliary ProUniversity of Minho. fessor in the Department of Industrial Electronics,

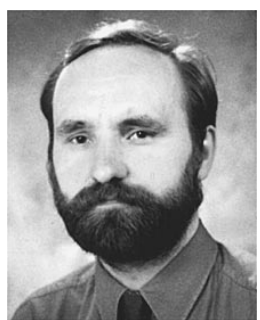

Marian Bartek (S'91-M'95) received the M.Sc. degree (cum laude) in electrical engineering in 1988 from the Electrical Engineering Department, Slovak Technical University, Bratislava, Slovakia. His master's thesis dealt with liquid phase epitaxy of InGaAsP quaternary layers for optoelectronic applications. He received the Ph.D. degree from the Department of Electrical Engineering, Delft University of Technology, The Netherlands, in 1995. $\mathrm{His} \mathrm{Ph} . \mathrm{D}$. research was on selective epitaxial growth for smart silicon sensor applications

After completion of his military service in October 1989, he continued this research at Slovak Technical University as a Research Assistant. In August 1991, he joined the Electronic Instrumentation Laboratory, Department of Electrical Engineering, Delft University of Technology. Currently, he is a Research Fellow. His work deals with technological aspects of integrated silicon sensor systems.

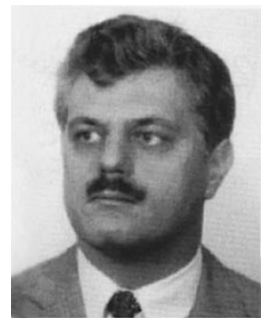

Reinoud F. Wolffenbuttel (S'86-M'89) received the M.Sc. degree in 1984 and the Ph.D. degree in 1988, both from the Delft University of Technology, The Netherlands. His thesis work dealt with the application of silicon to color sensing.

From 1986 to1993, he was an Assistant Professor, and since 1993 an Associate Professor, at the Laboratory of Electronic Instrumentation,Delft University of Technology. He is involved in instrumentation and measurement in general and on-chip functional integration of microelectronic circuits and silicon sensor, fabrication compatibility issues, and micromachining in silicon and microsystems in particular. In 1992-1993, he was a Visiting Scientist at the University of Michigan, Ann Arbor where he was involved in research on low-temperature wafer-to-wafer bonding. 